\title{
Estrés: manifestaciones en contextos académicos y análisis crítico del concepto
}

Stress: manifestations in school contexts and critical analysis of the concept

\author{
Ana Selene Hernández López ${ }^{a}$, Dulce Jatziri Oviedo Porras ${ }^{b}$, Deni Beelia Rodríguez Falcón ${ }^{c}$, \\ Karla Encarnación Martínez ${ }^{d}$, Jesús Cisneros-Herrera ${ }^{e}$, Gelacio Guzmán-Díaz $^{f}$
}

\begin{abstract}
:
In this paper, the concept of stress is explained, as well as a brief overview of some instruments measuring stress, and, at the end, a reflection about the use of this concept in psychology. Even if this is a non-exhaustive review of the concept and the instruments measuring it, it is supposed to be useful for those who are interested in stress, its measurement and its implication for the view of individual in society. It is necessary for psychologists to have a critical point of view regarding their concepts and not to lose sight of social aspects implied in the phenomena they study.
\end{abstract}

Keywords:

Stress, Academic Stress, Evaluation, Instruments

Resumen:

En este trabajo se expone el concepto de estrés, así como una reseña de algunos instrumentos que miden estrés y, por último, una reflexión acerca del uso de este concepto en psicología. Si bien esta presentación no es exhaustiva se espera que resulte inf ormativa para aquellos interesados en el estrés, en su medición y en sus implicaciones en la visión del individuo en sociedad. Es necesario que los psicólogos tengan una visión crítica acerca de sus conceptos, y no perder de vista los aspectos sociales de los fenómenos que estudian.

Palabras Clave:

Estrés, Estrés Académico, Evaluación, Instrumentos

\section{Estrés}

El término estrés es la castellanización del inglés stress, que a su vez deriva de los vocablos latinos strictus, que significa "apretado, estrecho", y stringere, apretar, constreñir (1). Así, en su origen etimológico, estrés hace referencia a una sensación somática y, por extensión, a una situación vital opresiva. Este origen emparente estrechamente al estrés con la ansiedad y la angustia, que provienen de un vocablo latín con el mismo significado, angere.

El estrés es la tensión psíquica, afectiva o física que experimenta el sujeto en su contexto y que tiene relación con las diferentes situaciones inmanejables que afectan directamente el control del individuo (2). El estrés hace que el sujeto reaccione a las situaciones y objetos estresantes, cada individuo tiene una reacción de forma diferente en función de sus características, sus redes de apoyo y recursos tanto materiales como simbólicos (3) (4). Los estímulos que provocan estrés pueden ser de naturaleza física que no pasan por la interpretación cognitiva, como el ruido excesivo constante o los lugares con mucha gente, o psicológica, cuando los estímulos son considerados estresantes después de una evaluación cognitiva, como los conflictos interpersonales, abuso físico o emocional/verbal, presenciar violencia doméstica o cambios en la vida. Sin embargo, la distinción no es tan clara, pues incluso con los estímulos puramente físicos

\footnotetext{
a Autor de Correspondencia, Universidad Autónoma del Estado de Hidalgo, https://orcid.org/0000-0003-0059-2336, Email: sel.hernandez13.95@gmail.com ${ }^{b}$ Universidad Autónoma del Estado de Hidalgo, https://orcid.org/0000-0003-1211-2520, Email: jatzirioviedoporras@gmail.com EUniversidad Autónoma del Estado de Hidalgo, https://orcid.org/0000-0001-6138-0762, Email: denibeelia97@ gmail.com ${ }^{\mathrm{d}}$ Universidad Autónoma del Estado de Hidalgo, https://orcid.org/0000-0002-0858-9409, Email: en347312@uaeh.edu.mx e Universidad Autónoma del Estado de Hidalgo, https://orcid.org/0000-0003-1370-8086, Email: jesus_cisneros@uaeh.edu.mx ${ }^{\mathrm{f}}$ Universidad Autónoma del Estado de Hidalgo, https://orcid.org/0000-0003-4869-8658, Email: gelacio_guzman@uaeh.edu.mx
} 
hay una evaluación que modera el nivel percibido es estrés que causan (5).

El estrés parece ser un rasgo distintivo de las sociedades industrializadas, y se ha asociado con problemas de salud, el bienestar y el rendimiento de las personas en distintos contextos sociales (6). La respuesta de la psicología ante este fenómeno ha sido proponer técnicas de afrontamiento del estrés por parte de los individuos, ubicando el problema en la valoración de los estímulos en vez de los estímulos en sí mismos.

El estrés transcurre en tres fases: de alarma, de resistencia y de agotamiento. Las cuales se llevan a cabo cuando el organismo debe prepararse a una nueva situación en la que gasta mayor energía para atacar, resistir o emprender la huida (7).

En la fase de alarma el organismo reconoce la presencia de una amenaza. La hipófisis activa las glándulas suprarrenales, produciendo la adrenalina noradrenalina y cortisol, que al llegar a la corriente sanguínea, generan una aceleración en algunos procesos y en otros un enlentecimiento. Los síntomas que aparecen en esta fase son: cefalea, fiebre, fatiga, dolor muscular, apnea, diarrea, malestar estomacal, inapetencia y falta de energía (8).

Durante la fase de alarma, el cuerpo se prepara para producir el máximo de energía que se da cuando enfrentamos una situación difícil, con los consecuentes cambios químicos. El cerebro, entonces, envía señales que activan la secreción de hormonas, que mediante una reacción en cadena provocan diferentes reacciones en el organismo, como tensión muscular, agudización de los sentidos, aumento en la frecuencia e intensidad de los latidos del corazón, elevación del flujo sanguíneo (9).

En la fase de resistencia se estabilizan los ajustes ante el estrés. Se desaparecen los síntomas de la fase anterior (alarma), en el exterior se percibe normalidad. El organismo logra enfrentar eficazmente el estresor, y disminuye la resistencia ante otros (8).

En la fase de agotamiento la activación, la tensión, los estímulos y demandas estresantes no disminuyen, el nivel de resistencia termina por agotarse, apareciendo de nuevo la alarma y en consecuencia los problemas tanto físicos como psicológicos. Esta fase provoca debilidad, se descansa mal, aparece sensación de angustia y deseo de huida (9).

Los síntomas que presentan el sujeto esta frente a una situación estresante son:

A nivel cognitivo: En este caso los pensamientos son continuos y se sobre analizan. La persona no encuentra solución a los conflictos que lo aquejan por más mínimos que sean. Por ejemplo, pensamientos ansiógenos y catastrofistas, dificultad para concentrarse, o dificultad para recordar, ansiedad, decrecimiento de la creatividad, rechinar de dientes, sudoración profusa, cefalea tensional, sensación de mareo, sensación de sofoco, dificultad para tragar, dolor de estómago, etc. (10)(11).

A nivel fisiológico: En este caso se observa presencia de enfermedades a nivel físico que van desde la sudoración, taquicardia, tensión, sensación de ahogo hasta dolores de cabeza, enfermedades estomacales e incluso infartos. También se pueden presentar conductas relacionadas como ingesta elevada de alcohol, depresión, agresividad, miedo, morderse las uñas, fumar, llanto, tendencia suicida o cambios en los hábitos alimenticios (10) (11).

A nivel motor: es entendido como las respuestas perceptibles que presenta el sujeto para hacer frente al estrés y evitar experimentar las consecuencias del estrés. Por ejemplo: contracciones musculares, necesidad de hidratación, estreñimiento, cefaleas, comezón, agotamiento, micción, mareos, vómitos (11).

\section{Estrés en el contexto escolar}

El estrés se presenta en múltiples situaciones, como los escenarios escolares. Las escuelas están pensadas para que los alumnos adquieran nuevas habilidades, confianza en sí mismos, creen relaciones con pares y adultos distintos de sus padres, y se preparen para la vida adulta. Sin embargo, rara vez ocurre esto (12).

Los principales factores que ocasionan estrés, en un estudiante por ejemplo, son (13):

- Falta de tiempo o tiempo limitado para poder cumplir con las actividades académicas.

- Sobrecarga académica, de estudio, de tareas o de trabajo escolares.

- Realización de un examen.

- Exposición de trabajos en clase.

- Realización de trabajos obligatorios para aprobar la asignatura.

- Exceso de responsabilidad por cumplir las obligaciones escolares.

- El tipo de trabajo que le piden los profesores.

- Mantener un buen rendimiento o promedio académico.

- La evaluación de los profesores.

\section{Instrumentos diagnósticos de estrés}

A continuación se presenta una relación no exhaustiva de algunos de los instrumentos utilizados para la evaluación del estrés académico con el objetivo de orientar a aquellos interesados en el diagnóstico.

Inventario SISCO

El inventario tiene como objetivo reconocer las características del estrés que suelen acompañar a los estudiantes. Se conforma de 47 ítems divididos en 46 ítems de tipo Likert y 1 ítem de tipo dicotómico; este instrumento permite obtener información sobre la 
presencia, intensidad y frecuencia del estrés, así como de sus tres componentes sistémico procesuales: estresores, síntomas y estrategias de afrontamiento (14).

En la escala SISCO, la intensidad hace referencia a identificar el nivel de estrés, es decir, que tan estresados se encuentran los alumnos; por otro lado la frecuencia permite saber los síntomas o reacciones ante al estímulo estresor y si existe la presencia del uso de estrategias de afrontamiento, es decir, cómo es que los alumnos se enfrentan durante situaciones estresantes (14).

Inventario de Estrés Académico (IEA)

Se compone de 21 ítems de tipo Likert; este instrumento permite plantear situaciones estresantes que puede padecer un estudiante universitario. Se utiliza una escala de respuesta tipo Likert con 5 opciones, en la que 1 significa que la persona no tiene o siente nada de estrés y 5 significa que el estudiante presenta mucho estrés (15). Instrumento estrés escolar

El instrumento consta de dos partes. La primera parte utiliza la técnica Likert y la segunda, ítems de respuesta múltiple. Las preguntas evalúan la relación entre las variables de estrés con respecto al rendimiento académico, estrés, familia e institución educativa, el área social dentro del contexto escolar, la desmotivación hacia algunas materias, la comprensión de lo explicado en el aula, la dificultad de concentración y la respuesta física que experimentan los estudiantes en el momento en que se encuentran nerviosos o preocupados (16).

Smith Relaxation States Inventory 3 (SRSI-3)

Consta de 38 ítems de tipo Likert conformada por 3 escalas: 1.Relajación básica asociado a la relajación física, 2. El descanso, la renovación y la relajación mental y 3 . Energía positiva relacionado con la energía y afecto positivo (17).

\section{Comentarios finales}

Como se mencionó antes, el estrés parece ser un rasgo distintivo de las sociedades modernas, en las que las desigualdades se han acentuado más. Cada vez hay más personas con bajos ingresos, sus jornadas de trabajo, por lo tanto, se han alargado para poder cubrir los gastos básicos. De modo que las preocupaciones económicas son permanentes para un amplio sector de la población. La pobreza también obliga a muchas personas a vivir en condiciones de hacinamiento. En México, los riesgos de ser víctima de delitos o de las múltiples formas de violencia social también son motivo de zozobra. Los desastres naturales, como los ciclones o la actual pandemia de COVID-19, también constituyen una amenaza latente para ciertas zonas geográficas en especial, o para toda la población.

Así, en el mundo abundan estímulos que amenazan con poner en peligro la vida o la subsistencia de las personas, por lo que la presencia de estrés y angustia está plenamente justificada. Estrés y angustia son síntomas de un malestar generalizado cuyo origen está en la organización político-económica de las sociedades, en el que las abrumadoras mayorías se encuentran en condiciones desfavorables. En este sentido, el alivio del estrés y la ansiedad, no compete al psicólogo, sino a las autoridades, cuya supuesta función es crear condiciones de vida digna para todos los ciudadanos.

Sin embargo, los psicólogos aceptan la misión de reducir los niveles de estrés en las personas, de modo que se hacen cómplices, tal vez sin darse cuenta, de una visión del ser humano y del mundo en la que el individuo es responsable de lo que siente. De este modo, siempre queda el recurso perverso de culpar al individuo de su estrés, de su ansiedad, de su depresión, restándoles legitimidad.

Es importante que el psicólogo examine esta de manera crítica su papel ante los problemas de las personas, pues las prácticas más populares en la actualidad, como las terapias cognitivo-conductuales o los talleres de desarrollo humano, explotan la idea de que el "control" sobre los estados afectivos y las reacciones corporales es total. Autoestima, resiliencia $y$, más recientemente, gaudabilidad, forman parte de una serie de conceptos que desconocen el papel de lo social en el malestar psicológico de las personas.

\section{Referencias}

[1] Stress and Learning. (2020). En V. Zeigler-Hill y T. K. Shackelford, Encyclopedia of Personality and Individual Differences (pp. 32023205). Cham: Springer. doi: 10.1007/978-3-319-24612-3_302613

[2] Serrano, M. G. \& Flores, M. M. (2005). Estrés respuesta de afrontamiento e ideación suicida. Revista Psicología y salud, 15.

[3] Ortega, J. (2014). Estrés y evaluación psicológica: un acercamiento teórico relacionado al concepto de resiliencia. Anuario de investigaciones. 21. 297-302. Recuperado de http://www.redalyc.org/pdf/3691/369139994030.pdf

[4] Pereira, M. (2009). Una revisión teórica sobre el estrés y algunos aspectos relevantes de éste en el ámbito educativo. Revista educación. 33, (2). 171-190. Recuperado de http://www.redalyc.org/articulo.oa?id=44012058011

[5] Stress. (2011). En J. S. Kreutzer, J. DeLuca y B. Caplan (eds.), Encyclopedia of Clinical Neuropsychology (pp. 2390-2391). Nueva York: Springer Science/Business Media. doi: 10.1007/978-0-38779948-3

[6] Stress. (2014). En A. C. Michalos (ed.), Encyclopedia of Quality of Life and Well-Being Research (pp. 6355-6357). Dordrecht: Springer Science/Business Media. doi: 10.1007/978-94-007-0753-5

[7] Díaz, R., Aguilar, V. \& Santillán, R. (1999). Estrés y embarazo. Revista de la Asociación Dental Mexicana. 44(1), 27-31. Recuperado de: https://www.medigraphic.com/pdfs/adm/od-1999/od991f.pdf

[8] Gonzáles, M. y González, S. (2012). Estrés académico en el nivel medio superior. Revista electrónica en Ciencias sociales y humanas apoyadas por tecnologías. 1(2), 32-70. Recuperado de https://edusol.info/revista/index.php/cshat/article/view/51/45 
[9] Ávila, J. (2014). Estrés un problema de salud del mundo actual. Revista CON- CIENCIA. 2, (1). 115-124. Recuperado de http://www.revistasbolivianas.org.bo/pdf/rcfb/v2n1/v2n1_a13.pdf

[10] Taylor, S. E. (2007). Estrés y afrontamiento. Psicología de la salud. México: McGraw-Hill Interamericana.

[11] Camargo, B. S. (2010). Estrés, síndrome general de adaptación o reacción general de alarma. Revista Medico Científica. 17 (2) 78-86. Recuperado de: http://www.revistamedicocientifica.org/index.php/rmc/article/view/103 1637.

[12] Galloway, M., Pope, D. y Osberg, J. (2007). Stressed-Out StudentsSOS: Youth perspectives on changing school climates. En. D. Thiessen y A. Cook-Sather (eds.), International Handbook of Student Experience in Elementary and Secondary School (pp. 611-634). Nueva York: Springer.

[13] Barraza, A., Martínez J., Silva, J., Camargo E. \& Antuna, R. (2011) Estresores académicos y género. Un estudio exploratorio de su relación en alumnos de licenciatura, Visión Educativa IUNAES, 5(12), 33-43. Recuperado https://dialnet.unirioja.es/servlet/articulo?codigo $=4034740$

[14] Barraza, A. (2019). Estrés académico en alumnos de tres niveles educativos. Un estudio comparativo. Praxis investigativa redie, 11, (21), 149-163.

[15] Castillo, C., Chacón, T. \& Díaz, G. (2016) Ansiedad y fuentes de estrés académico en estudiantes de carreras de la salud. Investigación en Educación Médica. 5(20) 230-237.

[16] Sierra, O. (2015). Estrés escolar y empatía en estudiantes de bachillerato practicantes de Mindfulness. Cuadernos de Lingüística Hispánica, 26, 175-197.

[17] Amutio, A., Franco, C., Gázquez, J. \& Mañas, I. (2015). Aprendizaje y práctica de la conciencia plena en estudiantes de bachillerato para potenciar la relajación y la autoeficacia en el rendimiento escolar. Universitas Psychologica, 14, (2), 433-443. 